\title{
eBalticGrid - an interactive platform for the visualisation of results from a high-resolution operational Baltic Sea model
}

\author{
Jaromir Jakacki, Anna Przyborska, Artur Nowicki, Marcin Wichorowski \\ Institute of Oceanology of the Polish Academy of Sciences, Powstańców Warszawy 55, $81-712$ Sopot, Poland, e-mail: \\ jjakacki@iopan.gda.pl
}

\author{
Michał Przyborski, Michał Białoskórski, Cyryl Sochacki, Rafał Tylman \\ Technical University of Gdańsk, Academic Computer Centre TASK, Narutowicza 11/12, 80-233 Gdańsk, Poland
}

\begin{abstract}
In recent years, modelling has been one of the fastest growing fields of science. Ocean, ice and atmospheric models have become a powerful tool that has supported many scientific fields during the last few decades. Our work presents the new operational service - called eBalticGrid - implemented into the PLGrid Infrastructure (Dziekoński et al. 2014). The grid is based on three modelling tools - an ocean model (Parallel Ocean Program), an ice model (Community Ice Code) and an atmospheric model (Whether Research and Forecasting Model). The service provides access to 72-hour forecasts for the Baltic Sea area. It includes the physical state of the Baltic Sea, its ice cover and the main atmospheric fields, which are the key drivers of the Baltic's physical state. Unlike other services, this provides the additional three-dimensional fields of temperature, salinity and currents in the Baltic Sea. The models work in operational mode and currently one simulation per day is run. The service has been implemented mostly for researchers. Access to the results does not require any modelling knowledge. Therefore, the main interface between a user and the model results was designed as a portal providing easy access to the model's output. It will also be a very suitable tool for teaching students about the hydrology of the Baltic Sea. Data from the system are delivered to another operational system - SatBaltic (Woźniak et al. 2011). The development of an output format to be suitable for navigational software (GRIB files) and sharing via FTP is also planned.
\end{abstract}

Key words: multimodelling, Baltic Sea, forecasting, hydrodynamic model, ice model

Submitted 20 July 2016, revised 3 December 2016, accepted 9 February 2017

\section{Introduction}

The Polish Grid Infrastructure (PGI) is an IT platform developed to support scientific investigations by integrating experimental data and the results of advanced computer simulations carried out by geographically distributed research teams. The PGI has been built under the framework of three main projects. The most important was PLGrid - a pioneer project started in 2009. The results are still handled by the main Polish computing centres and many researchers continue using the results. Recently, the PL-Grid New Generation (PLGrid NG) project - a descendant of the PLGrid - has been also completed. As a result of that project, several computing services for new groups of researchers have been implemented. The eBalticGrid (EBG) is one of the new tools implemented under the PGI. Its main task is to visualise and present the physical state of the Baltic Sea, as calculated by implemented model sets.

The Baltic Sea, with an area of ca. $377000 \mathrm{~km}^{2}$ and a volume of about $22000 \mathrm{~km}^{3}$, is one of the largest brackish water basins in the world. It spans between $53^{\circ} \mathrm{N}$ to $66^{\circ} \mathrm{N}$ latitude and $10^{\circ} \mathrm{E}$ to $30^{\circ} \mathrm{E}$ longitude and occupies a basin formed by glacial erosion. Connected to the North Atlantic via the Danish straits, this shelf sea is enclosed by Denmark, Sweden, Finland, Russia, Estonia, Latvia, Lithuania, Poland and Germany. It is relatively shallow, with an average depth of $52 \mathrm{~m}$, and only $10 \%$ of its area is deeper than $100 \mathrm{~m}$. The maximum depth is $459 \mathrm{~m}$ and the deepest point is located at the Landsort Deep near the Swedish coast. Due to its complex coastline and topography, the Baltic Sea can be divided into several separate regions: Gulf of Bothnia, Bay of Bothnia, Gulf of Finland, Gulf of Riga, Baltic Proper, Danish Straits and Kattegat. The largest part is the Baltic Proper, containing over 50\% of the water volume. The Sea consists of many smaller topographical elements which are, despite their size, essential for the hydrological properties of the basin. It is exposed to the influence of a number of changing environmental conditions, such as weather characteristics, freshwater discharge from rivers and inflows from the North Sea. A large number of rivers have their estuaries in the Baltic Sea; this brings a substantial supply of freshwater 
and nutrients. On the other hand, the exchange of water with the North Sea through the Danish Straits is limited. This leads to a very strong, permanent vertical stratification with respect to water density and salinity. Thermal stratification can also be observed. However, the depth of thermocline and the characteristics of stratification change significantly over a year. The average salinity in the Baltic Sea is ca. 7 PSU. However, this is much lower further away from the Danish Straits and in the partly enclosed bays with major freshwater inflows. Salty and heavier seawater flowing from the North Sea propagates near the bottom, thus, salinity can reach much higher values in deeper parts of the Baltic Proper and near the Danish Straits. In winter a considerable part of the Baltic Sea is covered by ice. The average annual maximum coverage reaches ca. $45 \%$ of its area. Due to its specificity Baltic Sea is a habitat for many freshwater as well as marine species, so it is a unique area for in-situ experiments, remote measurements and modelling (Osinski 2007).

In recent decades, modelling in physical oceanography (and, obviously, not only in that field) has become a powerful numerical tool providing a wide image of the three-dimensional physical state of the sea (Omstedt et al. 2014). Developments in computer technology resulted in the development of operational modelling systems. However, many of these systems are not available to the public and there are only a few systems that present results from operational models that cover the whole Baltic Sea. Most systems are limited to meteorological data, because it is important for commercial use. Examples are:

- http://yr.no/ (a service provided by Norwegian Meteorological Institute and the Norwegian Broadcasting Corporation; only meteorological data for the Baltic Sea are shown);

- http://meteo.pl/ (Interdisciplinary Modelling Centre at Warsaw University; only atmospheric data and Baltic Sea waves are presented);

- http://model.ocean.univ.gda.pl/ (University of Gdańsk, ecohydrodynamic model limited to the South Baltic area);
- http://deep.iopan.gda.pl/CEMBaltic/new_lay/index.php (Institute of Oceanology at the Polish Academy of Sciences (IOPAS), operational model focused on the Baltic Sea ecosystem (Dzierzbicka-Głowacka et al. 2013b).

As mentioned above, most of the systems present the meteorological state. Additionally, Baltic Sea ice conditions are presented as maps, not as results from an operational model (only IOPAS provides ice cover as an operational product).

The EBG (http://ebaltic.plgrid.pl) is a recently deployed operational platform that provides modelling results mostly for scientists. The platform is based on three models, namely atmosphere, ice and ocean. All of these models require High Performance Computing knowledge, a lot of experience in ocean-atmosphere-ice modelling and, additionally, Fortran or $\mathrm{C}$ programming skills. The complicated structure of the system prompted the idea of implementing such a service into the PLGrid Infrastructure, which would make it possible for researchers to have online access to the model's results without any knowledge about modelling and the output data structure. The platform provides access to the model results spread over time and space.

\section{Description of the eBaltic service}

The implemented platform consists of two main parts. The first one, which will be described here, is the model sets. The second one is the portal (working as a GUI) developed for end users. The block diagram of the service is presented in Fig. 1. A single step of the system can be simplified to the following sequence of actions:

- Importing atmospheric boundary conditions from the Global Forecasting System (GFS).

- Running an atmospheric model (72-hour integration mixed hindcast and forecast).

- Preparing atmospheric data for a coupled ice-ocean model.

- Importing boundary conditions for a coupled ice-ocean model.

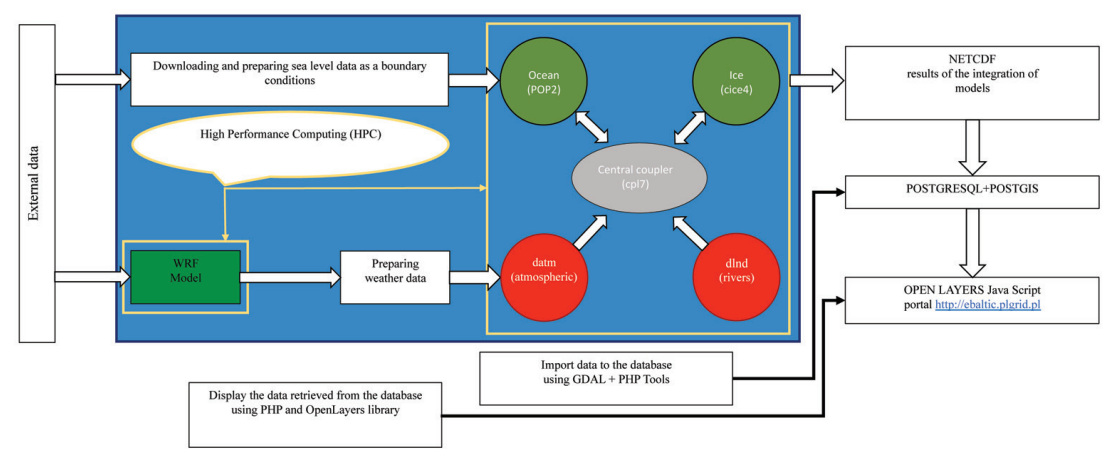

Fig. 1. Block diagram of the implemented service 
- Running the ice-ocean model (72-hour integration).

- Importing data to the database.

The main core of the platform is marked as the blue frame in Fig. 1 and it consists of five main components designated as red and green objects (models). The colour red was chosen for components called data models (datm and dind stand for atmospheric data model and land data model, respectively). This means that it is not an active model; these components only take data from external sources (for example from files or other models) and send it to the active components (marked as green rectangles). The main active components, a coupled ice-ocean model as one element and an atmospheric model as a separate component, are described below in separate subsections. The end user interface (portal) which is shown on the right side of the block diagram (Fig. 1) is also described in a separate section.

\subsection{Atmospheric model}

The forecast model used in the implemented system is the Weather Research and Forecasting Model - WRF (Skamarock et al. 2008). The WRF model is a next-generation mesoscale Numerical Weather Prediction (NWP) system designed to serve both operational forecasting and atmospheric research needs. The WRF model incorporates advanced numeric and data assimilation techniques, multiple relocatable nesting capability and improved physics, particularly for the treatment of convection and mesoscale precipitation systems.

The WRF model is a limited area, non-hydrostatic, primitive equation model with multiple options for various physical parameterisation schemes. More details regarding physics options are given in (Kumar et al. 2012). The horizontal resolution of the atmospheric model is $7 \mathrm{~km}$. Data needed for the ice-ocean model are saved every hour and interpolated on the ice-ocean grid after the integration is finalised. 72-hour simulation is performed on one node (12 cores) and takes less than 4 hours.

\subsection{Coupled ice-ocean model of the Baltic Sea}

The coupled ice-ocean model is the regional adaptation of the Community Earth System Model, or CESM (Craig et al. 2012; Kay et al. 2015) for the Baltic Sea. The main part - the ocean model - is based on the Los Alamos National Laboratory (LANL) Parallel Ocean Program, or POP (Smith, Gent 2004), evolved from the (Semtner 1974) global ocean model with added free surface formulation (Killworth et al. 1991). It is a z-level coordinate, general circulation ocean model (GCM) that solves 3-dimensional primitive equations for stratified fluid, using hydrostatic and Boussinesq approximations. Numerically, the model computes spatial derivatives in the spherical coordinates using the finite difference technique. The placement of model variables in the horizontal direction is an Arakawa B-grid (Arakawa, Lamb 1977). The barotropic equation is solved using a preconditioned conjugate gradient solver (PCG) (Hu et al. 2013) and cantered differencing represents the advection scheme. A biharmonic operator was chosen as a horizontal mixing parameterisation and a simple K-profile parameterisation to cover vertical mixing. We also used the equation of state introduced by (McDougall et al. 2003).

The ocean model is coupled through the "flux coupler" (also called cpl7) (Craig et al. 2005, 2012) with the sea ice model (Community Ice CodE - CICE model) (Craig et al. 2014). The CICE uses an elastic-viscous-plastic ice rheology (Hunke, Dukowicz 1997; Hunke 2001). The Los Alamos CICE model is the result of an effort to develop a computationally efficient sea ice component for a fully coupled atmosphere-ice-ocean-land global climate model. It was designed to be compatible with the POP for use on massively parallel computers. CICE has several interacting components: a thermodynamic model (Bitz, Lipscomb 1999) that computes local growth rates of snow and ice due to vertical conductive, radiative and turbulent fluxes, along with snowfall; a model of ice dynamics which predicts the velocity field of the ice pack based on a model of the material strength of the ice; a transport model that describes advection of the ice area concentration, ice volumes and other variables of the state; and a ridging parameterisation that transfers ice among thickness categories based on energetic balances and rates of strain (Lipscomb et al. 2007). The CICE also has multiple thickness categories and ice thickness distribution evolves over time.

The horizontal resolutions of POP and CICE are identical and equal to ca. $2,3 \mathrm{~km}$. The vertical resolution of the ocean model is 5 metres for the whole Baltic Sea. Initial conditions of the hydrodynamic model have been prepared based on salinity and temperature from a climatological data set for the Baltic Sea (Jansen et al. 1999), the model bathymetry has been interpolated from high-resolution spherical grid topography (Seifert et al. 2001). The spin-up time was 24 years (it was limited by the availability of sea level data for the lateral boundary). The model integration is performed on 8 nodes (96 cores), and the simulation time depends on ice cover - it usually takes less than one hour.

\subsection{Lateral boundary and real time implementation}

The model needs lateral boundary conditions to provide the correct solutions for the areas where the model domain 
ends. The Baltic Sea is connected to the North Sea through the Kattegat and Skagerrak. For proper representation of the flow through the Danish Straits (DS) it is sufficient to reproduce the sea level over the DS and it is also necessary to set salinity and temperature values at the boundary area (Stevens 1990; Meier, Kauker 2003). The operational model calculations are supported by two external sea level data sources. These are both observations and forecasts. The observational data are aggregated by BOOS (Baltic Operational Oceanographic System) from 24 stations along the Baltic coast (of which 22 operate and send data in real time) and transferred via FTP protocol for scientific purposes. There are quality-checking procedures applied to reduce gross errors. Within the eBaltic frame those data are downloaded, processed and stored in a local relational database. The database interface is organized as an SQL query via the ODBC connection, which may be easily implemented using scripting languages (PHP, Perl) as well as other tools and libraries compatible with a MySQL driver. It is possible to customise the query, setting time frame and the spatial domain (station Id - in our model we use observational data from a mareograph located in Gothenburg). The observational data are updated every hour. The forecast data are generated as HIROMB (Funkquist, Ljungemyr 1997) results provided by the Swedish Meteorological and Hydrological Institute (SMHI) service and are generated every 6 hours. Each forecast dataset is downloaded locally and stored in a relational database as forecast data. The data may be retrieved by specification of timeframe of the forecast, spatial extent and time of the forecast generation.

The running time of the whole system is governed by the software utility Cron - a time-based job scheduler.

\section{End user interface (eBaltic portal)}

As mentioned above, the interface used by a standard user for obtaining access to the model's results is the portal. To get full access to the forecast, a user has to register in the PLGrid Infrastructure (http://www.plgrid.pl/) and apply for access to the eBaltic service. The portal is also open to the public with very limited access to the results (only surface data are shown and the main functionalities of the portal, such as sections, zooming and animations, are disabled). Access to the service for registered users is granted automatically via OpenID service (an open standard and decentralised protocol by the non-profit OpenID Foundation which allows users to be authenticated by certain cooperating sites using a third party service - Wikipedia).

The results of the models are stored in the Network
Common Data Form (Netcdf) or, in the case of the atmospheric data, are converted into this format. Importing one output data set from the models is performed for the portal in three main steps (see the right side of Fig. 1). In the beginning, the NETCDF files are converted into an SQL query using Geospatial Data Abstraction Library (GDAL) tools. Then, the data in raster format are loaded into the database PostgreSQL with the Spatial and Geographic Objects (PostGIS) extension. In the last step the data are adapted to the geographical projection used by the portal. The portal uses two JavaScript libraries - OpenLayers v 3.3, responsible for geo-mapping of the images, and Highcharts, which generates 2D charts. By using raster data storage it can quickly generate images in the format of png files and prepare the raw data necessary for charts showing variation in time or space.

The portal presents output fields in three main groups naturally related to mediums (only 3D fields are denoted, all the other fields are 2D):

- the sea (POP):

- temperature (3D);

- salinity (3D);

- sea currents (3D);

- barotropic stream function;

- sea level;

- ice cover (CICE):

- ice concentration;

- ice thickness;

- frazil ice;

- ice drift;

- atmosphere (WRF):

- temperature at 2 metres above sea level;

- humidity at 2 metres above sea level;

- pressure reduced to sea level;

- sea surface net thermal radiation;

- sea surface net solar radiation;

- wind speed and direction at $10 \mathrm{~m}$ above sea level;

- total precipitation;

- snowfall.

It is also possible to zoom in on an interesting area and make a section between many points. For $3 \mathrm{D}$ fields it is possible to select the model level (depth). The eBaltic portal allows the creation of animations for any given $2 \mathrm{D}$ field (which means that animations for any field combined with a vertical section are allowed). Example portal screenshots are shown in Fig. 2:

a) standard view after logging in (2,5-metre temperature of the whole Baltic Sea);

b) zoomed sea surface salinity (at a depth of 2,5 m);

c) vertical section of temperature;

d) zoomed 10-metre wind speed. 

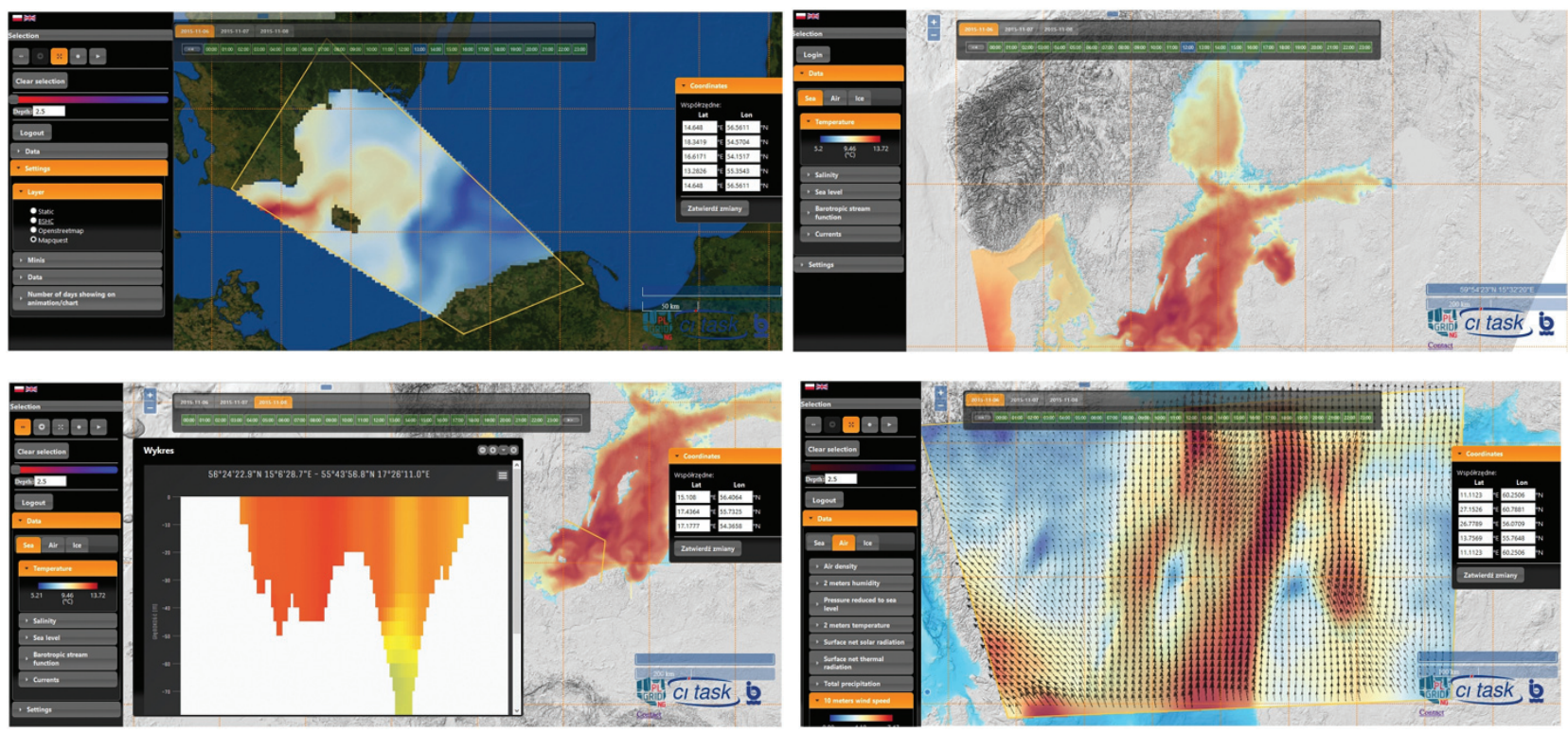

Fig. 2. Examples of different views of the eBalitic service

The system allows the use of different background maps, which is also shown in Fig. 2. Furthermore, it is possible to select a specific hour for the visible data (the system default physical state is displayed for the local time).

\section{Integration with the PLGrid infrastructure}

As far as the above aspects of the calculation for environmental forecasts are concerned, there are at least three issues to discuss. Firstly, all the data should be calculated as soon as possible, in order for it to be suitable for further use. Secondly, all calculations should be performed on reliable resources in order to ensure that they start at a fixed time and last for approximately the same period of time. Thirdly, all the archived data should be saved to an efficient and safe storage system. All these issues can be resolved with the proper system architecture, stable software, firm and efficient hardware, and qualified personnel managing all these components and providing user support. The required hardware, with an excellent network connection, is provided by the PL-GRID part of the infrastructure, located at the Academic Computer Centre in Gdańsk (CI TASK).

To achieve a reasonable calculation time, jobs are run in a parallel mode using MPI on 96 processor cores (8 cluster nodes connected with the InfiniBand network). With these resources, a forecast for 24 hours is calculated within 1 hour. After the calculation is finished, all the data are uploaded to the database. However, before beginning ice-ocean integration, the atmospheric model has to make a forecast simulation, which takes 12 cores (one node) and consumes about 4 hours of wall time.
The data upload is done by translation of files from the model calculation in netCDF format to the text SQL command executed on an HPC (High Performance Computation) server, as described in the previous section of this paper. A single SQL command generated in this way occupies up to $300 \mathrm{MB}$. During the upload, one process on the HPC server and 4 processes on the database server are occupied $100 \%$. The time needed for uploading $24 \mathrm{~h}$ data sets to the database is as long as the time needed for the calculation. Work on the optimisation of the process is under way. In order to have new data presented at constant intervals, calculation tasks are run on the HPC server under a job queuing system with a special resource reserve taken for the EBG forecast calculation. The architecture of the EBG portal, including the data flowchart, is shown in Fig. 3.

All the data - current and archived - are stored on the DB server. All logged-in users have access to the current and archived data.

Currently, all calculations are performed on the GaleraPlus HPC cluster installed at the CI TASK. This cluster is made up of Xeon 6-core two-processor nodes with 16 GB

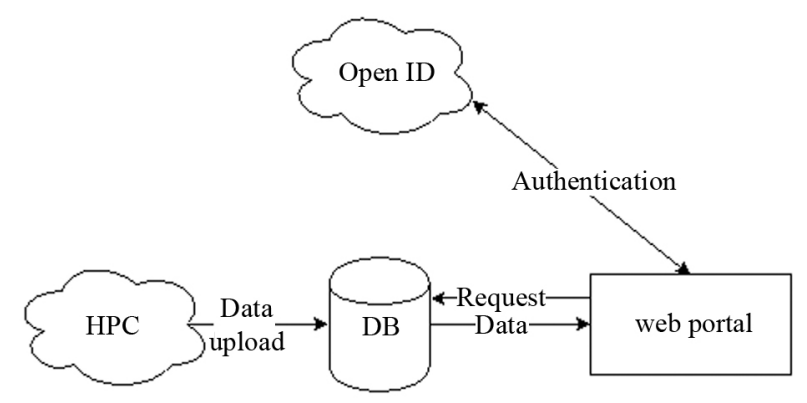

Fig. 3. Flowchart of the eBalticGrid structure 
of RAM connected to the InfiniBand QDR network in the fat-tree topology. For the EBG there is a dedicated link to the database. The database is hosted on a 4-core node with $4 \mathrm{~GB}$ of RAM.

Another service provided by the EBG is providing an archive for the data on the physical state of the Baltic Sea. The archived data are used in studies of environmental evolution or for the comparison of forecasts while developing new calculation systems and models. The safe storage of data is the most important aspect of the service.

The applicability of the forecast data on the physical state of the environment depends on their accuracy, validity and availability. If the data are unavailable at fixed times, this will cause problems for EBG users. Similar difficulties will appear when the data are inaccurate or invalid.

\section{Conclusions and future plans}

It is expected that the data published in the EBG will be used by scientists and autonomic systems. The platform fulfilled all the PLGrid requirements and was successfully implemented in the Infrastructure.

The prediction of ice cover and its thickness (but also other physical parameters such as wind speed and direction or waves) can be widely used both in economic and social activities. In the economy, forecasts and the state of the ice cover allows for the planning of tasks associated with clearing waterways and the organisation of harbour operations connected with the preparation of vessels to protect ports from complete freezing and the stoppage of operation. Furthermore, this includes carrying out work required to secure infrastructure, planning fishing activities, scheduling and organising sports events, training related to iceboats as well as utilizing the icy waterfront as a tourist attraction. Valid and reliable ice cover data will also allow for early warnings of thin ice. Knowing and forecasting the state of ice cover will provide additional support for maritime rescue systems in winter.

The implementation of other models into the platform (for example, a wave model) is planned, as is the extension of the forecast to up to 7 days and the introducing of basic statistical analysis. However, the current hardware resources are insufficient for such a development. Therefore, extension of the hardware platform is planned, with all the simulations to be run on the newest HPC cluster available on the CI TASK - Tryton equipment with double Xeon E5-v3 12-core nodes and with 192GB RAM. The database will be hosted on a dedicated server of similar parameters connected with the HPC cluster via a $10 \mathrm{GE}$ link. Using such hardware resources should make it pos- sible to double the speed of operation. All the data will be stored on the Hitachi HUS-150 storage system.

\section{Acknowledgements}

This work was made possible thanks to the PLGrid NG project (POIG.02.03.00-12-138/13), co-funded by the European Regional Development Fund as part of the Innovative Economy programme, including a special purpose grant from the Polish Ministry of Science and Higher Education.

\section{Bibliography}

Arakawa A., Lamb V.R., 1977, Computational design of the basic dynamic processes of the UCLA general circulation model, Methods in Computational Physics, 17, 173-265

Bitz C.M., Lipscomb W.H., 1999, An energy-conserving thermodynamic model of sea ice, Journal of Geophysical Research, 104 (C7), 15669-15677, DOI: 10.1029/1999JC900100

Craig A., Jacob R., Kauffman B., Bettge T., Larson J., Ong E., He H., 2005, CPL6: The new extensible, high-performance parallel coupler for the Community Climate System Model, The International Journal of High Performance Computing Applications, 19 (3), 309-328

Craig A.P., Mickelson S., Hunke E.C., Bailey D.A., 2015, Improved parallel performance of the CICE model in CESM1, International Journal of High Performance Computing Applications, 29 (2), 154-165

Craig A.P., Vertenstein M., Jacob R., 2012, A new flexible coupler for earth system modelling developed for CCSM4 and CESM1, The International Journal of High Performance Computing Applications, 26 (1), 31-42

Dziekoński P., Klajn F., Flis Ł., Lasoń P., Magryś M., Oziębło A., Rowicki R., Stolarek M., Bartkiewicz D., Zawadzki M., Pospieszny M., Mikołajczak R., Brzeźniak M., Meyer N., Samson M., 2014, National distributed high performance computing infrastructure for PL-Grid users, [in:] eScience on distributed computing infrastructure, M. Bubak, J. Kitowski, K. Wiatr (eds.), Lecture Notes in Computer Science Series, 8500, 16-33, DOI: 10.1007/978-3-319-10894-0_2

Dzierzbicka-Głowacka L., Jakacki J., Janecki M., Nowicki A., 2013a, Activation of the operational ecohydrodynamic model (3D CEMBS) - the hydrodynamic part, Oceanologia, 55 (3), 519-541, DOI: 10.5697/oc.55-3.519

Dzierzbicka-Głowacka L., Janecki M., Nowicki A., Jakacki J., 2013b, Activation of the operational ecohydrodynamic model (3D CEMBS) - the ecosystem module, Oceanologia, 55 (3), 543-572, DOI: 10.5697/oc.55-3.543

Funkquist L., Ljungemyr P., 1997, Validation of HIROMB during 1995-1996, SMHO Oceanografi, 67, 10 pp. 
Hu Y., Huang X., Wang X., Fu H., Xu S., Ruan H., Xue W., Yang G., 2013, A scalable barotropic mode solver for the Parallel Ocean Program, [in:] Euro-Par 2013 Parallel Processing, F. Wolf, D an Mey (eds.), Lecture Notes in Computer Science Series, 8097, 739-750, DOI: 10.1007/978-3-642-40047-6_74

Hunke E.C., 2001, Viscous-plastic sea ice dynamics with the EVP model: Linearization issues, Journal of Computational Physics, 170 (1), 18-38, DOI: 10.1006/jcph.2001.6710

HunkeE.C., Dukowicz J.K., 1997, An elastic-viscous-plastic model for sea ice dynamics, Journal of Physical Oceanography, 27, 1849-1867, DOI: 10.1175/1520-0485(1997)027<1849:AEV $\mathrm{PMF}>2.0 . \mathrm{CO} ; 2$

Jansen F., Schrum C., Backhaus J.O., 1999, A climatological data set of temperature and salinity for the Baltic Sea and the North Sea, Deutsche Hydrographische Zeitschrift, 51, 5, DOI: $10.1007 / \mathrm{BF} 02933676$

Kay J.E., Deser C., Phillips A., Mai A., Hannay C., Strand G., Arblaster J., Bates S., Danabasoglu G., Edwards J., Holland H., Kushner P., Lamarque J.F., Lawrence D., Lindsay K., Middleton A., Munoz E., Neale R., Oleson K., Polvani L., Vertenste M., 2015, The Community Earth System Model (CESM) Large Ensemble Project: A community resource for studying climate change in the presence of internal climate variability, Bulletin of the American Meteorological Society, 8, 1333-1349, DOI: 10.1175/BAMS-D-13-00255.1

Killworth P.D., Stainforth D., Webb D.J., Paterson S.M., 1991, The development of a free-surface Bryan-Cox-Semtner ocean model, Journal of Physical Oceanography, 21, 13331348, DOI: 10.1175/1520-0485(1991)021<1333:TDOAFS > 2.0.CO;2

Kumar P., Bhattacharya B.K., Pal P.K., 2012, Impact of geostationary satellite INSAT-3A CCD generated vegetation fraction on regional model forecast, Agricultural and Forest Meteorology, 168, 82-92,

Lipscomb W.H., Hunke E.C., Maslowski W., Jakacki J., 2007, Ridging, strength, and stability in high-resolution sea ice models, Journal of Geophysical Research, 112 (C3), 1-18, DOI: 10.1029/2005JC003355

McDougall T.J., Jackett D.R., Wright D.G., Feistel R., 2003, Accurate and computationally efficient algorithms for potential temperature and density of seawater, Journal of Atmospheric and Oceanic Technology, 20, 730-741
Meier M., Kauker F., 2003, Modeling decadal variability of the Baltic Sea: 2. Role of freshwater inflow and large-scale atmospheric circulation for salinity, Journal of Geophysical Research, 108 (C11), DOI: 10.1029/2003JC001799

Omstedt A., Elken J., Lehmann A., Leppäranta M., Meier H.E.M., Myrberg K., Rutgersson A., 2014, Progress in physical oceanography of the Baltic Sea during the 20032014 period, Progress in Oceanography, 128, 139-171, DOI: 10.1016/j.pocean.2014.08.010

Osinski R., 2007, Simulation of dynamic processes in the Baltic Sea based on integrated ocean-ice model, PhD. Thesis, Institute of Oceanology Polish Academy of Sciences

Seifert T., Tauber F., Kayser B., 2001, A high resolution spherical grid topography of the Baltic Sea - revised edition, Baltic Sea Science Congress, November 25-29, Stockholm, Sweden, poster 147

Semtner A.J., 1974, A general circulation model for the World Ocean, Department of Meteorology Technical Report, University of California Los Angeles, 99 pp.

Skamarock W., Klemp J., Dudhia J., Gill D., Barker D.M., Duda M.G., Huang X.Y., Wang W., Powers J., 2008, A description of the advanced research WRF. Version 3, NCAR Technical Note, NCAR/TN-475+STR, available at http://www2. mmm.ucar.edu/wrf/users/docs/arw_v3.pdf (data access 23.02.2017)

Smith R., Gent P., 2004, Reference manual for the Parallel Ocean Program (POP), Los Alamos National Laboratory, New Mexico

Stevens D.P., 1990, On open boundary conditions for three dimensional primitive equation ocean circulation models, Geophysical \& Astrophysical Fluid Dynamics, 51, 103-133

Woźniak B., Bradtke K., Darecki M., Dera J., Dudzińska-Nowak J., Dzierzbicka-Głowacka L., Ficek D., Furmańczyk K., Kowalewski M., Krężel A., Majchrowski R., Ostrowska M., Paszkuta M., Stoń-Egiert J., Stramska M., Zapadka T., 2011, SatBałtyk - A Baltic environmental satellite remote sensing system - an ongoing project in Poland. Part 1: Assumptions, scope and operating range, Oceanologia, 53 (4), 897-924, DOI: $10.5697 /$ oc.53-4.897 